\title{
Carreira e bem-estar subjetivo no ensino superior: Determinantes pessoais e situacionais
}

\author{
Maria Odilia Teixeira \\ Cátia João Costa \\ Faculdade de Psicologia da Universidade de Lisboa, Lisboa, Portugal
}

\section{Resumo}

No modelo de Bem-Estar de Lent e Brown de 2006, é analisada a relação entre bem-estar subjectivo e carreira, considerando variáveis pessoais, educacionais e contextuais. Os participantes são 255 estudantes de Psicologia (52\%) e Saúde (48\%) (média etária 20,40; 86\% mulheres) que responderam às Escalas Satisfação na Carreira, Autoeficácia Geral, Desenvolvimento e Bem-Estar, Exigência e Responsividade Parental, Big Five-10 e Adaptação ao Ensino Superior. Os resultados da regressão múltipla linear mostram como preditores de bem-estar: satisfação na carreira, percepção dos factores pessoais de ajustamento ao curso, autoeficácia, percepção de responsividade materna, extroversão e amabilidade. Esta pesquisa identifica dimensões contributivas do bem-estar dos estudantes, que são significativas na intervenção, e esclarece o significado da carreira no âmbito da Psicologia Positiva. Palavras-chave: psicologia positiva, autoeficácia, personalidade, estilos parentais, ajustamento ao curso

Abstract: Career and subjective well-being in higher education: personal and situational determinants

In the 2006 Lent and Brown Well-being model, the relationship between subjective well-being and career is analyzed, considering personal, educational and contextual variables. The participants were 255 students of Psychology (52\%) and Health (48\%) (average age 20.40, 86\% women) who responded to the Career Satisfaction, General Self-Efficacy, Development and Well-Being, Parental Responsibility and Demanding, Big Five-10 and Adaptation to Higher Education Scales. The results of the linear multiple regression show as predictors of well-being: career satisfaction, perception of the personal factors of course adjustment, self-efficacy, perception of maternal responsibility, extroversion and kindness. This research identifies contributory dimensions of student well-being, which are significant in intervention, and clarifies the meaning of the career in the field of Positive Psychology.

Keywords: positive psychology, self-efficacy, personality, parental styles, course adjustment

\section{Resumen: Carrera y bienestar subjetivo en la enseñanza superior: determinantes personales y contextuales}

En el modelo de Bienestar de Lent y Brown de 2006, se analiza la relación entre bienestar subjetivo y carrera, considerando variables personales, educativas y contextuales. Los participantes fueron 255 estudiantes de Psicología (52\%) y Salud (48\%) (promedio de edad 20.40, 86\% mujeres), que respondieron las Escalas de Satisfacción en la Carrera, Autoeficacia General, Desarrollo y Bienestar, Exigencia y Responsividad Parental, Big Five-10 y adaptación a la enseñanza superior. Los resultados de la regresión múltiple lineal muestran como predictores de bienestar: satisfacción en la carrera, percepción de los factores personales de ajuste al curso, autoeficacia, percepción de la responsividad materna, extroversión y amabilidad. La investigación identifica dimensiones contributivas del bienestar de los estudiantes que son significativas en la intervención y aclara el significado de la carrera en el ámbito de la Psicología Positiva.

Palabras clave: psicología positiva, autoeficacia, personalidad, estilos parentales, ajuste al curso

\footnotetext{
${ }^{1}$ Endereço para correspondência: Faculdade de Psicologia, Universidade de Lisboa, Alameda da Universidade, 1649-013, Lisboa, Portugal.E-mail: moteixeira@psicologia.ulisboa.pt
} 
Desde há décadas que o domínio da Psicologia da Carreira extravasa as áreas específicas da profissão ou formação e se entrecruza com os domínios da saúde e bem-estar (e.g., Zunker, 2008). Os modelos atuais do aconselhamento da carreira (e.g., Amundson, HarrisBowlsbey, \& Niles, 2014; Gysbers, Heppner, \& Johnston, 2014; Savickas, 2013) caracterizam-se pela natureza integradora das principais teorias da Psicologia Vocacional (e.g., Holland, 1997; Lent, Brown, \& Hackett, 1994; Super, 1990) e introduzem propostas procedentes da Psicologia Positiva (Seligman \& Csikszentmihalyi, 2000) e dos modelos de Aconselhamento Pessoal (e.g., Cormier, Nuris, \& Osborne, 2013; Rogers, 1961). Na Psicologia Positiva e no Aconselhamento são questões centrais o significado da vida, as condições do desenvolvimento das forças positivas do funcionamento psicológico e o trabalho como espaço de desenvolvimento e de bem-estar (Dik, Duffy, O’Donnell, Shim, \& Steger, 2015; Robertson, 2015). A inserção da Psicologia Positiva no âmbito do Aconselhamento da Carreira vem, por um lado, posicionar o eixo da intervenção vocacional na visão holística da pessoa e, por outro lado, acentuar o papel da formação e do trabalho nos modelos de bem-estar (Connolly \& Myers, 2003; Robertson, 2015). A convergência destas linhas paradigmáticas da Psicologia enfatiza o agenciamento pessoal, que coloca na capacidade das pessoas a construção da vida, nomeadamente no domínio da carreira. Esta será a melhor resposta da intervenção nas atuais circunstâncias de risco social, em que a vida se tornou menos previsível, regulada, estável e ordenada (Duarte, 2017).

No curso da vida, também a trajectória no ensino superior representa um mundo de oportunidades em termos de desenvolvimento pessoal, uma fase estruturante da identidade dos jovens adultos (Erikson, 1968) e um contexto de contínuo fluir (flow) no sentido dado por Mihaly Csikszentmihalyi (2004) de que fluir é um estado de envolvimento numa actividade com objetivos bem definidos. O estado de fluir emerge em situações que envolvem desafios e elevado padrão de competências como atenção, concentração e foco (Dik et. al., 2015), sendo as experiências académicas e profissionais fontes significativas para a percepção do fluir vital. O fluir representa o sentimento da sintonia percebida entre corpo e mente, o equilíbrio entre desafios e competências de enfrentamento (Csikszentmihalyi, 1990). A exploração e experimentação dos estados de fluir representam ainda um dos propósitos da intervenção vocacional; os objetivos da carreira operam como agentes motivacionais, que atribuem significado à vida (Ramos, Paixão, \& Simões, 2011) e atuam como preditores do bem-estar (Ramos, 2016; Rogers, Creed, \& Searle, 2012). Verducci e Gardner
(2006) introduziram o conceito de bom trabalho, que faz a ponte entre a Psicologia Positiva e a carreira. Este conceito traduz possibilidades de as instituições otimizarem condições de desenvolvimento de competências técnicas e éticas, que proporcionam emoções positivas e atribuições positivas à vida no trabalho.

A intervenção educacional de carreira é plena de potencialidades que fomentam a consciência pessoal e dos ambientes de formação e de trabalho, numa procura permanente de encontrar significados, envolvimento, harmonia e bem-estar. As intervenções no ensino superior visam potenciar fluir, florescimento, desenvolvimento, satisfação e realização, em que o jovem adulto pode consolidar o seu estilo de vida pessoal. Na acepção de Adler, (1967), entende-se estilo de vida como movimento único do indivíduo em relação aos objectivos autopropostos e aos seus ideais. O conceito florescimento foi introduzido por Keyes (2002), e, refere-se ao modo como as pessoas desenvolvem potencialidades e vivem emoções positivas, de forma a atingirem padrões de qualidade de vida individual e coletiva (Seligman, 2011). A carreira é um dos domínios da vida mais significativos para planejar, estabelecer objectivos e criar significado e envolvimento na própria vida (Connolly \& Myers, 2003).

\section{Modelo de Bem-Estar Subjetivo}

Lent e Brown (2006) organizaram um modelo de Bem-Estar para os domínios académico e profissional, que operacionaliza a relação entre Psicologia Positiva e Psicologia da Carreira através da relação do bem-estar e da satisfação na carreira. De acordo com o princípio holístico da Psicologia Positiva, o modelo de Lent e Brown (2006) é contextualizado na dinâmica da vida. A satisfação da carreira e bem-estar são determinados por variáveis sociais, cognitivas, comportamentais e de personalidade, sendo a relação destas variáveis mediada por processos cognitivos de que fazem parte objetivos, expetativas e crenças de autoeficácia (Lent \& Brown, 2006). Os estudos com o modelo de bem-estar em estudantes do ensino superior evidenciam tanto o papel dos objectivos (Lent, et al., 2005; Ramos, Paixão, \& Simões, 2011) como dos interesses (Lent, Taveira, \& Costa-Lobo, 2012) na prossecução do bem-estar, e, por sua vez, bem-estar tem impato na aprendizagem e no desenvolvimento de competências académicas (Ramos, Paixão, \& Simões, 2011).

O bem-estar subjectivo é normalmente associado à tradição filosófica de hedonismo, sendo a procura de bem-estar, prazer e felicidade o bem supremo da vida humana. Myers, Sweeney e Witmer (2000) definem bem-estar como um modo de vida orientado para otimizar 
saúde física e psicológica, em que corpo, mente e espírito estão integrados para alcançar uma vida plena no plano individual mas também na comunidade social e no seio da natureza. $\mathrm{O}$ conceito de bem-estar subjectivo associa componentes cognitivos e emocionais como afetos, crenças de autoeficácia, autonomia, competências emocionais e intelectuais, adaptação e experiências (Diener, Suh, Lucas, \& Smith, 1999).

No modelo de Bem-Estar de Lent e Brown (2006), as crenças de autoeficácia são os principais mecanismos cognitivos do agenciamento pessoal (Bandura, 1986), responsáveis pelo comportamento, circunscrição das escolhas e perseverança para ultrapassar obstáculos. A análise das crenças de eficácia remete necessariamente para aprendizagem em meio social, com destaque para os contextos da família, escola e pares que veiculam oportunidades, modelos, expetativas e feedback nas diferentes situações de aprendizagem, de natureza social e académica (Ahn, Usher, Butz, \& Bong, 2016; Laranjeira \& Teixeira, 2016; Teixeira \& Ferreira, no prelo). No âmbito da aprendizagem social, consideram-se as experiências, a modelagem, as emoções, os estados fisiológicos e a persuasão social na formação das crenças de autoeficácia, no seio da relação triádica entre pessoa, comportamento e situação (Bandura, 1986). A pesquisa evidencia que a autoeficácia académica é bom preditor de sucesso, da satisfação académica (Ojeda, Navarro, \& Flores, 2011), das aspirações e escolhas de carreira (Lopes \& Teixeira, 2012) e do bem-estar (Ramos, 2016), sendo a autoeficácia também determinada pelas aspirações, desempenhos e expetativas familiares e sociais (Teixeira \& Ferreira, no prelo).

O modelo de Bem-Estar subjetivo de Lent e Brown (2006) considera ainda os traços de personalidade como fator facilitador de desempenhos, satisfação e bem-estar. O conceito de personalidade é entendido como organização consistente e estável dos principais traços de personalidade nos cinco grandes fatores propostos por McCrae e Costa (1996): neuroticismo, extroversão, abertura à experiencia, amabilidade e conscienciosidade. Estes traços de personalidade são fortes preditores do bem-estar, sendo a estabilidade emocional e a extroversão normalmente associados aos sentimentos de alegria, bem-estar e emoções positivas (Rogers, et. al., 2012). No domínio da Psicologia Vocacional, o modelo de personalidade de J. Holland (1997) atribui particular enfoque à congruência entre caraterísticas de personalidade e ambiente. A percepção pessoal sobre a congruência desempenha um papel chave na motivação e bem-estar, e determina a realização profissional e satisfação na carreira (Holland, 1997). A tomada de consciência das características pessoais desencadeia sentimentos de realização, plenitude, persistência e exploração da carreira (Lent, et. al., 2005).

Entre os fatores contextuais que influenciam o bem-estar, a família estabelece um conjunto de variáveis proximais que tem sido estudado no âmbito dos contextos educacionais e de carreira (e.g., Hill \& Wang, 2015; Teixeira \& Ferreira, no prelo), e da saúde e bem-estar (e.g., Hutz \& Bardagi, 2006). A pesquisa tem demonstrado que para além das variáveis demográficas (e.g., nível socioeconómico, habilitações académicas e naturalidade da família) (Hill \& Wang, 2015; Lopes \& Teixeira, 2012), as práticas e atitudes parentais, que constituem o estilo de parentalidade, têm influência no bem-estar da criança e do jovem (Hutz \& Bardagi, 2006). O estilo da parentalidade é um dos principais agentes de desenvolvimento na criança e a sua influência ocorre ao longo da vida, nomeadamente na adolescência e no jovem adulto (Teixeira \& Ferreira, no prelo; Hill \& Wang, 2015). No âmbito da parentalidade, a pesquisa tende a diferenciar os papéis dos progenitores (Costa, Teixeira, \& Gomes, 2000; Teixeira, Bardagi, \& Gomes, 2004), sendo a influência da mãe mais forte nas emoções, crenças e valores (Teixeira \& Bardagi, 2016; Teixeira \& Laranjeira, 2016) e a influência do pai mais sentida nos níveis de aspiração e nas tomadas de decisão (Christofides, Hoy, Milla, \& Stengos, 2015; Teixeira \& Ferreira, no prelo). As diferenças de género também emergem no modo como são percepcionados os padrões de parentalidade, sendo as raparigas mais sensíveis à percepção dos padrões de exigência parental, do que os rapazes (Bardagi \& Teixeira, 2015; Teixeira \& Bardagi, 2016).

\section{Método}

Considerando a literatura sobre bem-estar e carreira, esta investigação visa clarificar a relação entre carreira e Psicologia Positiva, bem como aprofundar o modelo de bem-estar subjectivo proposto por Lent e Brown (2006). $\mathrm{O}$ senso de bem-estar é examinado de acordo com variáveis de carreira, operacionalizadas na percepção dos factores relacionados com o curso (ajustamento pessoal, reconhecimento social, envolvimento, relacionamento), satisfação na carreira, traços de personalidade, autoeficácia geral e percepção do padrão educacional parental. Seguindo as premissas do modelo de Bem-Estar subjectivo, são hipóteses da pesquisa: (a) existe relação positiva entre satisfação na carreira e senso de bem-estar; (b) as variáveis de carreira (consciência dos factores pessoais de ajustamento ao curso, percepção de recompensas sociais e materiais e envolvimento no curso) determinam o bem-estar; (c) os traços de personalidade de extroversão e amabilidade e a percepção do relacionamento positivo no curso contribuem para o senso de bem-estar; 
(d) as crenças de autoeficácia determinam o senso de bem-estar e (e) a percepção das atitudes de responsividade do pai e da mãe influencia o senso de bem-estar.

\section{Participantes}

A amostra é constituída por 255 estudantes do ensino superior em Portugal, que frequentam o $2^{\circ}$ ano do curso de Psicologia (52\%) da Universidade de Lisboa e cursos da área da Saúde (48\%) do Instituto Politécnico de Leiria. Nos cursos de Saúde estão incluídos Terapia Ocupacional (15\%), Terapia da Fala (7\%), Enfermagem (11\%) e Fisioterapia $(14 \%)$. As idades variam entre 19 e 31 anos $(M=20,40$, DP $=2,09$ ), e as raparigas representam $84 \%$ da amostra.

\section{Instrumentos}

O caderno de questionários é composto pelo Questionário de Adaptação ao Ensino Superior, Escala de Desenvolvimento e Bem-Estar (Flourishing Scale), Escala de Satisfação na Carreira (Career Satisfaction Scale), Big 5 Inventory-10 (BFI - 10), Escala de Autoeficácia Geral (General Self-Efficacy Scale) e Escalas de Exigência e Responsividade Parental.

O Questionário de Adaptação ao Ensino Superior foi elaborado por Costa e Teixeira (2016) com o propósito de avaliar a percepção dos factores de ajustamento ao curso (e.g., $\mathrm{O}$ curso corresponde às minhas competências/ capacidades) e factores de adaptação (e.g., Relação com os colegas). É um questionário de auto-relato, com os conteúdos distribuídos por 15 itens em 4 subescalas que focam a percepção dos factores pessoais de ajustamento ao curso (4 itens), relacionais (3 itens), recompensas sociais e materiais (3 itens) e envolvimento no curso (5 itens). Estas subescalas correspondem aos factores ortogonais derivados de procedimentos em componentes principais com rotação varimax, numa amostra do ensino superior (Costa \& Teixeira, 2016). A resposta é dada numa escala de Likert de cinco pontos. Os dados relativos à precisão indicam coeficientes entre 0,58 e 0,74 (Costa \& Teixeira, 2016), situando-se os mais baixos no limite do aceitável, de acordo com Maroco e Garcia-Marques (2006). O questionário inclui ainda a identificação sociodemográfica dos participantes (e.g., sexo, idade, curso, escola).

A Escala de Desenvolvimento e Bem-estar (Flourishing Scale) (Diener, Wirtz, Biswas-Diener, Tov, Kim-Prieto, Choi, \& Oishi, 2009) avalia o julgamento da própria vida, em áreas como sucesso pessoal, relacionamentos interpessoais, autoestima, sentido de vida e otimismo (e.g., Vivo uma vida com propósito e significado). A Escala é de auto-relato, unidimensional, contém oito itens, sendo a resposta dada em escala de Likert de sete pontos. Existe uma grande diversidade de pesquisa com esta escala, salientando-se estudos com amostras do Brasil que confirmam a natureza unidimensional da medida e o elevado grau de consistência interna, cujo alfa de Cronbach se situa em 0,84 (Fonseca, Nascimento, Barbosa, Vione, \& Gouveia, 2015). Esta escala foi adaptada pelas autoras da pesquisa à língua portuguesa de Portugal.

A Escala de Satisfação na Carreira (Career Satisfaction Scale) de Greenhaus, Parasuraman e Wormley, construída em 1990, (Hofmans, Dries, \& Pepermans, 2008) avalia a visão pessoal sobre progressão da própria carreira de vida, incluindo objetivos e visão de sucesso (e.g., Estou satisfeito(a) com os progressos que fiz para atingir os meus objetivos materiais). Esta escala é unidimensional, contém cinco itens com resposta em escala de Likert de cinco pontos. Em diferentes amostras, esta medida tem revelado um grau elevado de consistência interna, com coeficientes alfa à volta de 0,80 (Beutell \& Wittig-Berman, 1999). A adaptação desta escala para o contexto português foi realizada por uma das autoras.

O Big Five Inventory (BFI - 10) de Rammstedt e Jonh (2007) corresponde a uma das formas reduzidas do Inventário Big Five de McCrae e Costa (1996). Este instrumento contém 10 itens divididos pelos cinco principais traços da estrutura da personalidade -neuroticismo, extroversão, amabilidade, conscienciosidade e abertura à experiência. A resposta é dada numa escala de Likert de cinco pontos. De acordo com Rammstedt e John (2007), os dois itens de cada uma das escalas foram selecionados pelas respetivas propriedades psicométricas, e no seu conjunto as escalas Extroversão, Conscienciosidade e Neuroticismo revelam bons índices de consistência interna $(0,99-0,82) \mathrm{e}$ de estabilidade temporal $(0,83-0,74)$. Em amostras de estudantes universitários ingleses e alemães foi identificada uma solução fatorial de cinco fatores com correspondência às cinco dimensões de personalidade anteriormente enunciados (Rammstedt \& John, 2007).

A Escala de Autoeficácia Geral (General Selfefficacy Scale) de Ralf Schwarzer e Matthias Jerusalem (1995) avalia a percepção de capacidade pessoal para resolver situações quotidianas com obstáculos. A escala é unidimensional, contém 10 itens (e.g., Consigo resolver sempre os problemas difíceis, se me esforçar), sendo resposta dada em escala de Likert de quatro pontos. A medida tem revelado indicadores positivos de consistência interna, com alfas de Cronbach próximos de 0,80 (Schwarzer, 2017), situando-se em 0,85 numa amostra de estudantes no Brasil (Sbicigo, Teixeira, Dias, \& Dell'Aglio, 2012). A pesquisa tem indicado existir uma relação entre os resultados da escala com otimismo e 
satisfação no trabalho (Schwarzer, 2017), bem como com autoestima e expetativas de futuro (Sbicigo, Teixeira, Dias, \& Dell'Aglio, 2012). Esta escala foi adaptada pelas autoras da pesquisa à língua portuguesa de Portugal.

A Escala de Exigência e Responsividade Parental foi adaptada da versão elaborada para a população do Brasil (Costa, et. al., 2000; Teixeira, et. al., 2004), designada Escala de Percepção de Exigência e Responsividade. Esta escala foi construída com base nos trabalhos de Lamborn (1991) e avalia a percepção dos jovens sobre responsividade e exigência dos pais. A escala contém 24 itens para a mãe e outros 24 para o pai, distribuídos pelas escalas de Exigência e Responsividade. A resposta é dada numa escala de Likert de cinco pontos. Os dados da versão do Brasil revelam uma estrutura bifatorial da medida com correspondência às escalas Exigência e Responsividade materna e paterna (Formiga, 2010; Teixeira, et. als., 2004). Os alfas de Cronbach indicam um grau elevado de consistência interna, sendo os coeficientes próximos de 0,80 (Teixeira, et. al., 2004). Nas amostras de adolescentes portugueses, os coeficientes alfa são superiores a 0,80 , e identifica-se uma estrutura factorial que corresponde também às escalas teoricamente previstas de Exigência e Responsividade (Bardagi \& Teixeira, 2015). Outros dados de validade indicam a relação dos estilos parentais com emoções positivas (Hutz \& Bardagi, 2000), empenhamento académico e confiança e envolvimento na carreira (Teixeira \& Bardagi, 2016), e percepções de auto-estima e de auto-eficácia (Costa, et., al., 2000; Teixeira, et. al., 2004; Teixeira \& Laranjeira, 2016). Em termos de validade intercultural, os dados em amostras de estudantes do ensino médio do Brasil e de Portugal evidenciam convergência quer na consistência interna quer na estrutura interna da medida (Teixeira, Bardagi, \& Teixeira, 2016).

\section{Procedimentos}

Os procedimentos deontológicos foram atendidos na aprovação do projeto de pesquisa pela Comissão de Deontologia da Faculdade de Psicologia da Universidade de Lisboa, na assinatura do consentimento informado, previamente à aplicação, e no pedido de autorização aos autores de todos os questionários. Nas escalas Desenvolvimento e Bem-estar e Autoeficácia Geral as traduções foram feitas por duas pessoas bilingues e uma retroversão por uma terceira pessoa, visto não existir versão portuguesa dos instrumentos, que fosse conhecida das investigadoras. Também na Escala de Exigência e Responsividade Parental houve necessidade de adaptar algumas expressões à língua portuguesa de Portugal. Os questionários foram aplicados de forma colectiva, em contexto sala de aula, com um tempo de aplicação de aproximadamente de 20 minutos.

Nas análises estatísticas, procedeu-se, em primeiro lugar, à estimativa das características das distribuições dos resultados, bem como do grau de consistência interna das diferentes medidas na presente amostra, recorrendo-se ainda às estatísticas inferenciais (correlações) a fim de avaliar o grau de associação das variáveis. Na sequência, efectuou-se uma regressão múltipla linear para analisar relações de causualidade, e identificar variáveis preditoras de bem-estar dos alunos da amostra. $\mathrm{O}$ tratamento dos dados foi realizado com recurso ao software estatístico IBM SPSS Statistics 22.

\section{Resultados}

\section{Distribuições, precisão e correlações}

A Tabela 1 apresenta, para o conjunto das medidas, as estatísticas descritivas das distribuições dos resultados e os coeficientes alfa de Cronbach. Na maioria das escalas, os índices da amplitude e os desvios-padrão indicam variabilidade dos resultados e sensibilidade das medidas às diferenças individuais. Contudo, nas escalas do Inventário de Personalidade (BFI - 10), os dados evidenciam assimetria das distribuições, e o mesmo acontece na dimensão relacional do Questionário de Adaptação ao Ensino Superior. A maioria dos alfas de Cronbach são satisfatórios, com excepção para as Escalas do Inventário de Personalidade, cujos coeficientes variam entre 0,46 e 0,68. Estes coeficientes são baixos (Maroco \& Garcia-Marques, 2006) e derivam certamente do fato de cada subescala incluir apenas 2 itens. Rammstedt e John (2007) também referem fracos índices de consistência interna nas escalas Amabilidade e Abertura à Experiência. Na presente pesquisa, estas duas escalas e ainda Conscienciosidade indicam coeficientes alfa ao redor de 0,46-0,48. No Questionário de Adaptação ao Ensino Superior, a Escala Envolvimento no Curso também regista o coeficiente de 0,58 , indicando também um fraco nível de consistência interna (Maroco \& Garcia-Marques, 2006).

A Tabela 2 apresenta a matriz de correlações dos resultados dos seis instrumentos. Na análise, consideram-se os coeficientes superiores a $|0,30|$, de acordo com o critério de que este valor representa uma correlação de força média, segundo alguns autores (e.g., Steele, Andrews, \& Upton, 2012, p. 67). Os resultados da escala de Desenvolvimento e Bem-estar (escala 5) associam-se com os das dimensões percepção dos Factores Pessoais de Ajustamento ao Curso $(0,41)$, Satisfação na Carreira $(0,54)$, Autoeficácia $(0,34)$, Percepção de Responsividade Materna $(0,41)$ e Extroversão $(0,33)$. Para além da 
associação com Bem-Estar, a Escala de Satisfação na

Carreira (escala 6) tem um coeficiente de 0,38 com os resultados da escala percepção dos Factores Pessoais de Ajustamento ao Curso.

Tabela 1

Amplitudes, médias, desvios-padrão e coeficientes alfa de Cronbach

\begin{tabular}{|c|c|c|c|c|}
\hline Escalas & Amplitude & Média & $\mathrm{DP}$ & Alfa \\
\hline \multicolumn{5}{|l|}{ Ajustamento e Adaptação ao Ensino Superior } \\
\hline Dimensão ajustamento pessoal ao curso & $8-20$ & 16,29 & 2,17 & 0,74 \\
\hline Dimensão de recompensas sociais e materiais & $6-15$ & 10,52 & 2,10 & 0,74 \\
\hline Dimensão relacional & $6-15$ & 11,50 & 1,61 & 0,62 \\
\hline Dimensão envolvimento no curso & $9-25$ & 16,11 & 2,44 & 0,58 \\
\hline Escala de desenvolvimento e bem-estar & $8-56$ & 42,59 & 6,72 & 0,88 \\
\hline Escala de satisfação na carreira & $5-35$ & 18,11 & 3,69 & 0,85 \\
\hline Escala de autoeficácia Geral & $11-61$ & 28,44 & 5,18 & 0,84 \\
\hline \multicolumn{5}{|l|}{ Estilos Parentais } \\
\hline Exigência materna & $14-60$ & 39,83 & 8,61 & 0,85 \\
\hline Responsividade materna & $14-62$ & 50,03 & 10,08 & 0,95 \\
\hline Exigência paterna & $12-59$ & 35,77 & 10,26 & 0,89 \\
\hline Responsividade paterna & $12-66$ & 46,25 & 12,26 & 0,94 \\
\hline \multicolumn{5}{|l|}{ Big Five Inventory $(\mathrm{BFI}-10)$} \\
\hline Extroversão & $2-10$ & 5,92 & 1,87 & 0,68 \\
\hline Amabilidade & $3-10$ & 7,41 & 1,50 & 0,46 \\
\hline Conscienciosidade & $2-10$ & 7,01 & 1,64 & 0,46 \\
\hline Neuroticismo & $2-10$ & 5,82 & 1,85 & 0,54 \\
\hline Abertura à Experiência & $3-10$ & 7,14 & 1,68 & 0,48 \\
\hline
\end{tabular}

Tabela 2

Correlações entre os resultados dos seis instrumentos

\begin{tabular}{|c|c|c|c|c|c|c|c|c|c|c|c|c|c|c|c|c|}
\hline & 1 & 2 & 3 & 4 & 5 & 6 & 7 & 8 & 9 & 10 & 11 & 12 & 13 & 14 & 15 & $\overline{16}$ \\
\hline 1 & & & & & & & & & & & & & & & & \\
\hline 2 & $46 * *$ & & & & & & & & & & & & & & & \\
\hline 3 &, $35 * *$ &, $26^{* *}$ & & & & & & & & & & & & & & \\
\hline 4 & $27 * *$ &, $14^{*}$ &, $25^{* *}$ & & & & & & & & & & & & & \\
\hline 5 &, $41 * *$ &, $28^{* *}$ &, $25^{* *}$ &, $15^{*}$ & & & & & & & & & & & & \\
\hline 6 &, $38^{* *}$ & $18^{* *}$ &, $28^{* *}$ &, $20 * *$ &, $54^{* *}$ & & & & & & & & & & & \\
\hline 7 & $17 * *$ & $18^{* *}$ &, $18^{* *}$ & $25^{* *}$ &, $34^{* *}$ &, $25^{* *}$ & & & & & & & & & & \\
\hline 8 &, $16^{*}$ & ,06 &, $13^{*}$ &, 02 & 05 &, 06 &,- 01 & & & & & & & & & \\
\hline 9 & $23 * *$ & $20^{* *}$ & 08 & 03 &, $41 * *$ &, $25 * *$ &, 04 &, $25^{* *}$ & & & & & & & & \\
\hline 10 & 10 & 10 &, $14^{*}$ &,- 08 &, 01 & ,10 &,- 03 &, $58^{* *}$ &, $14^{*}$ & & & & & & & \\
\hline 11 & 06 & 11 & 09 & 03 &, $19^{* *}$ &, $18^{* *}$ & ,04 &, $27^{* *}$ &, $44^{* *}$ &, $55^{* *}$ & & & & & & \\
\hline 12 & 10 & $13^{*}$ &, $20 * *$ & 07 &, $33 * *$ &, 11 &, $22 * *$ &, 13 & $17^{* *}$ &, $15^{*}$ &, $19 * *$ & & & & & \\
\hline 13 &,- 01 &,- 01 & , 12 &,- 08 &, $22^{* *}$ & 03 &,- 04 & 07 &, 08 & 05 & ,09 &, $21 * *$ & & & & \\
\hline 14 &, 11 &,- 00 & 03 &, $14^{*}$ &, $17^{* *}$ &, $27 * *$ & $15^{*}$ &,- 01 &, $14^{*}$ &,- 10 & 02 &, $14^{*}$ &,- 02 & & & \\
\hline 15 &,- 01 & 11 & 06 & , 10 &, $17^{* *}$ & ,06 &, $31 * *$ & 04 &,- 07 & ,04 & 06 &, $15^{*}$ & 06 &,- 06 & & \\
\hline 16 & ,00 & ,00 &,- 02 &, 01 &, 05 & ,00 &, $27 * *$ &, 02 & 01 & ,01 &,- 07 & ,09 &, 10 &,- 01 & , 10 & \\
\hline
\end{tabular}

Nota: 1. Ajustamento pessoal ao curso, 2. Recompensas sociais e materiais, 3. Relacional, 4. Envolvimento no curso, 5. Escala de desenvolvimento e bem-estar, 6. Escala de satisfação na carreira, 7. Escala de autoeficácia Geral, 8. Exigência materna, 9. Responsividade materna, 10. Exigência paterna, 11. Responsividade paterna, 12. Extroversão, 13. Amabilidade, 14. Conscienciosidade, 15. Neuroticismo, 16. Abertura à Experiência 


\section{Regressão múltipla linear}

Procedeu-se a uma análise de regressão múltipla linear pelo método "enter", considerando-se como variável dependente o Bem-Estar e como variáveis independentes a Satisfação na Carreira, as percepções Ajustamento Pessoal ao Curso, Relacional, Recompensas Sociais e Materiais e Envolvimento no Curso do Questionário Ajustamento e Adaptação ao Ensino Superior, a Auto-eficácia Geral, a Exigência e Responsividade Parental e os factores de personalidade Neuroticismo, Extroversão, Amabilidade, Conscienciosidade e Abertura à Experiência do Inventário de Personalidade (BFI - 10). Foram testados os pressupostos do modelo, nomeadamente o da distribuição normal, multicolinearidade, homogeneidade e independência dos erros (valor de Durbin-Watson $=2,18$ ), verificando-se que existem 20 outliers com potencial impacto na estimação da reta de regressão. Este dado foi obtido através dos resultados decorrentes da distância de Mahalanobis. Adicionalmente, o modelo de regressão foi estimado com e sem os valores extremos identificados para averiguar o seu impacto nos valores estimados. Apesar dos resultados serem semelhantes entre estes modelos, respeitou-se a indicação da distância de Mahalanobis apresentando-se os resultados para o modelo sem a inclusão dos outliers.

O modelo de regressão múltipla linear é responsável por uma parte significativa da variabilidade do Bem-Estar $\left(F(15,206)=17,97 ; p<0.001 ; R^{2}=0,58\right)$ e permite explicar o Bem-Estar Subjetivo com as variáveis preditoras Satisfação na Carreira $(\beta=0,25 ; t(14)=4,21 ; p<0,001)$, Ajustamento Pessoal ao Curso $(\beta=0,20 ; t(14)=3,42$; $p<0,01)$, Autoeficácia Geral $(\beta=0,20 ; t(14)=3,48$; $p<0,01)$, percepção da Responsividade Materna ( $\beta=$ $0,28 ; t(14)=4,73 ; p<0,001)$, Extroversão $(\beta=0,14$; $t(14)=2,75 ; p<0,01)$ e Amabilidade $(\beta=0,22$; $t(14)=4,47 ; p<0,001)$.

\section{Discussão}

Apesar das limitações atribuídas à própria amostra $\mathrm{e}$ às características psicométricas de algumas medidas, esta pesquisa proporciona indicadores que clarificam a relação entre Carreira e Psicologia Positiva, e aprofundam o modelo de bem-estar subjectivo de Lent e Brown (2006). A discussão dos resultados respeitantes ao Inventário de Personalidade (BFI - 10) é particularmente condicionada pelo baixo grau de consistência interna, principalmente revelada nas escalas Conscienciosidade $(0,46)$, Abertura à Experiência $(0,48)$ e Amabilidade $(0,46)$, sendo os coeficientes das duas últimas também relativamente fracas no estudo de Rammstedt e John (2007).
Estes dados estão associados ao fato do instrumento incluir apenas dois itens em cada escala, pois corresponde a uma forma reduzida de Inventário de Personalidade Big Five (Rammstedt \& John, 2007). A discussão dos resultados segue a ordem das hipóteses apresentadas para a pesquisa.

A H1 tende a ser confirmada pelas correlações moderadas, mas significativas, entre Bem-Estar e Satisfação na Carreira $(0,46)$ e Bem-Estar e percepção dos Factores Pessoais de Ajustamento ao curso $(0,41)$. Estas associações constituem indicadores da relação entre variáveis de carreira e bem-estar subjetivo, e são corroboradas por Connolly e Myers (2003) que enfatizam a relação entre trabalho e bem-estar como parte significativa dos modelos holísticos de bem-estar. No mesmo sentido, Robertson (2015) realça a importância do trabalho como contexto que dá significado à vida, que proporciona desenvolvimento e bem-estar. No aprofundamento da relação entre as variáveis da carreira e o bem-estar, a H2 também é parcialmente confirmada nos resultados da regressão múltipla linear. A dimensão de percepção dos Factores Pessoais de Ajustamento ao Curso é preditiva do Bem-Estar subjetivo dos alunos da amostra. No mesmo sentido, os dados de um estudo qualitativo de Teixeira e Gomes (2004) corroboram a importância atribuída pelos estudantes do ensino superior às características pessoais face à formação e à transição, sendo comprovado o poder preditivo dos interesses no ajustamento e bem-estar dos estudantes de Engenharia (Lent et. al., 2012). Todas estas evidências são confirmadas pelo princípio da congruência como condição para a satisfação, equilíbrio e bem-estar (Holland, 1997; Spokane, Meir, \& Catalano, 2000), e salientam o papel chave do autoconhecimento para o florescimento e o bem-estar (Dik, et al., 2015). Nesta amostra, a percepção de recompensas sociais e materiais e o envolvimento no curso não determinam significativamente o senso de bem-estar.

A H3 foi parcialmente confirmada. Os resultados da regressão múltipla linear confirmam extroversão e amabilidade como dimensões preditivas do bem-estar. Estes dados são comprovados pela relação anteriormente encontrada entre níveis de extroversão, sociabilidade, otimismo e adaptação ao ensino superior (Ntalianis, 2010), sendo a estabilidade emocional e a extroversão envoltas em emoções positivas que desencadeiam bem-estar (Rogers, et. al., 2012). Salienta-se assim a importância do fator do relacionamento pessoal nos níveis de bem-estar, tal como corroborado por Granado, Santos, Almeida, Soares e Guisande (2005) e Ramos (2016). Contudo, nos resultados da presente pesquisa, a percepção do fator pessoal na adaptação ao curso não é significativa nos níveis de 
bem-estar, ao contrário do que seria de esperar face aos resultados anteriores. Este resultado pode dever-se à relativa homogeneidade da amostra, que inclui maioritariamente mulheres e apenas estudantes das áreas de Psicologia e Saúde. Nestes grupos, normalmente é privilegiado o fator relacional pela larga maioria dos estudantes. De referir ainda que a escala de relacionamento revela um índice de precisão $(0,62)$ no limite da aceitação (Maroco \& GarciaMarques, 2006).

A H4 é confirmada também pelos resultados da regressão múltipla linear em que as crenças de autoeficácia surgem como bons preditores do bem-estar, tal como preconizam Lent, et. al., (2005). Estes dados são ainda corroborados pela correlação moderada entre os resultados autoeficácia e autoestima (Sbicigo, Teixeira, Dias, \& Dell'Aglio, 2012), correspondendo a autoestima a uma das emoções positivas do bem-estar subjectivo. Também no estudo de Teixeira e Gomes (2004), os estudantes do ensino superior revelaram uma associação entre senso de competência e envolvimento com a formação.

A H5 também foi parcialmente confirmada pelos resultados da regressão múltipla linear que explicitam o contributo preditivo da percepção Responsividade Materna para o bem-estar dos jovens adultos da amostra. Estes dados são confirmados pela importância das mães na educação e formação dos seus educandos (Ahn, et. al., 2016; Teixeira \& Bargagi, 2016), sendo também corroborados pelos estudos que diferenciam o papel das mães no suporte emocional e dos pais como modelos profissionais mais presentes nas decisões sobre continuidade dos estudos (Bardagi e Hutz, 2008; Teixeira \& Ferreira, no prelo). Estes resultados também confirmam a permanência da influência dos pais na vida do jovem adulto (Hill \& Wang, 2015), e de que a parentalidade responsiva incrementa nos jovens o desenvolvimento da autonomia e das competências para lidar com a vida e a carreira (Lamborn, 1991; Teixeira \& Bardagi, 2016).

\section{Limitações}

A amostra é de conveniência e inclui apenas alunos dos cursos de Psicologia e da área da Saúde de duas Escolas do Ensino Superior, com sobre representação do sexo feminino. As investigações futuras procurarão amostras mais heterogéneas e introduzir outras variáveis relacionadas com a vida académica e social dos estudantes do ensino superior. Salientam-se ainda os baixos níveis de consistência interna de algumas medidas, particularmente da Escala BFI-10 e do Questionário de Adaptação ao Ensino Superior, com claras implicações nos resultados.

\section{Considerações Finais}

Esta pesquisa tende a evidenciar o papel das variáveis da carreira como fontes de desenvolvimento e bem-estar dos estudantes de Psicologia e Saúde, nomeadamente no que concerne ao senso de satisfação com o percurso autoproposto, à consciência das qualidades pessoais relacionadas com o curso, ao senso de autoeficácia e ainda às características de personalidade ligadas às emoções positivas nos relacionamentos interpessoais. Estes indicadores possibilitam inferências de que a carreira representa espaço de possibilidades de florescimento de potencialidades, de fluir vital e confiança pessoal e interpessoal, e, consequentemente permitem aprofundar o modelo de Bem-Estar de Lent e Brown (2006). Os dados tendem a clarificar a relação entre os domínios da Psicologia da Carreira e da Psicologia Positiva e a explicar o sentimento de bem-estar dos estudantes de Psicologia e de Saúde desta amostra. No futuro será pertinente relacionar estes dados com variáveis do domínio académico, tais como qualidade das aprendizagens, sucesso escolar e abandono escolar.

Estes fatores que explicam o desenvolvimento e bem-estar a nível individual e de grupo devem ser considerados no planejamento das intervenções de carreira e são incentivadores à criação e programação dos serviços de apoio ao estudante por parte das instituições do ensino superior. Os objetivos da intervenção da carreira ultrapassam a visão tradicional de "encaixe pessoa ambiente" reformulando-se em propósitos de autoconhecimento reflexivo e estabelecimento de objetivos e projetos que atribuam significado à vida e às aprendizagens. Alguns autores sugerem (e.g., Robertson 2015) a necessidade de os profissionais ajudarem os indivíduos a integrarem o trabalho e a vida, para que possam desenvolver envolvimento e significado pessoal no trabalho. As abordagens multidisciplinares e holísticas colocam o trabalho e a formação como um dos principais papéis e tarefas de vida na procura de satisfação e bem-estar (Myers, Sweeney, \& Witmer, 2000). Destaca-se a relevância do enquadramento da carreira na vida e dos modelos que atribuem centralidade à consciência e gestão das características pessoais (Duarte, 2017), nomeadamente no campo da personalidade e autoeficácia.

Os resultados chamam também à atenção sobre os padrões educacionais da família que acompanham a vida e o senso de bem-estar do jovem adulto do ensino superior. Esta questão remete para uma visão desenvolvimentista não só sobre a carreira, mas também sobre as influências contextuais, nomeadamente da parte familiar. Sublinha-se a necessidade de atuar com a família, em qualquer fase da vida, de forma a desenvolver padrões de parentalidade 
responsiva, que promovam autonomia, satisfação e bem-estar, e ainda atitudes que enfrentem o trabalho como fonte de desenvolvimento e realização pessoal.

Nas atuais circunstâncias de precaridade social e numa exigência de qualidade e sucesso das aprendizagens, as instituições do ensino superior devem priorizar a criação de estruturas de apoio funcional e emocional que acompanhem o estudante ao longo do ensino superior, e os profissionais devem ser competentes em estabelecer pontes entre os domínios académicos, social, emocional e vocacional, nas intervenções ao longo da vida, nomeadamente no ensino superior. A investigação que se centra nos indicadores de bem-estar deve, no nosso entender, responder às preocupações das instituições (e.g., sucesso académico, abandono escolar) e co-responsabilizar investigadores e organizações, no sentido de identificar e implementar condições que favoreçam o florescimento das capacidades dos alunos e das instituições.

\section{Referências}

Adler, A. (1967). A Ciência da Natureza Humana (6th ed.). São Paulo: Companhia Editora Nacional.

Ahn, H. S., Usher, E. L., Butz A., \& Sonf, M. (2016). Cultural differences in the understanding of modeling and feedback as sources of self-efficacy information. British Journal of Educational Psychology, 86(1), 112-136. DOI: 10.1111/ bjep.12093.

Amundson, N. E., Harris-Bowlsbey, J. E., \& Niles, S. G. (2014). Essential Elements of Career Counseling: Processes and Techniques ( $3^{\text {rd }}$ ed.). Pearson (E-Book).

Bardagi, M. P. \& Hutz, C. S. (2008). Apoio parental percebido no contexto da escolha inicial e da evasão de curso universitário. Revista Brasileira de Orientação profissional, 9(2), 31-44. Recuperado em 27 de setembro de 2017, de http://pepsic.bvsalud.org/pdf/rbop/v9n2/v9n2a05.pdf

Bardagi, M. P. \& Teixeira, M. O. (2015, julho). Psychometrical properties of Portuguese and Brazilian versions of the Parental Demandingness and Responsiveness Scale: An intercultural study. Comunicação apresentada no $13^{\text {th }}$ European Conference on Psychological Assessment. Universidade de Zurique: Zurique.

Bandura, A. (1986). Social foundations of thought and action: A social cognitive theory. Englewood Cliffs. NJ: Prentice-Hall.

Beutell, N.J. \& Wittig-Berman (1999). Predictors of work-family conflict and satisfaction with family, job, career, and life. Psychological Reports, 85, 893-903. DOI: 10.2466/pr0.1999.85.3.893.

Christofides, L. N., Hoy, M., Milla, J., \& Stengos, T. (2015). Grades, Aspirations, and Postsecondary Education Outcomes. Canadian Journal of Higher Education, 45(1), 48-82. Recuperado em 27 de setembro de 2017, de http://journals.sfu. ca/cjhe/index.php/cjhe/article/viewFile/184203/pdf_1

Connolly, K. \& Myers J. (2003). Wellness and mattering: The role of holistic factors in job satisfaction. Journal of Employment Counseling, 40(4), 152-160. DOI: 10.1002/j.2161-1920. 2003.tb00866.x

Cormier, S., Nuris, P. S., \& Osborn, C. J. (2013). Interviewing and Change Strategies for Helpers ( $7^{\text {th }}$ ed.). Belmont, CA: Brooks Cole.

Costa, C. J. \& Teixeira, M. O. (2016, junho). Carreira e bem-estar subjetivo no ensino superior: determinantes pessoais e situacionais. IX Simpósio Nacional de Investigação em Psicologia. Universidade do Algarve. Faro.

Costa, F. T., Teixeira, M. A. P., \& Gomes, W. B. (2000). Responsividade e exigência: Duas escalas para avaliar estilos parentais. Psicologia: Reflexão e Crítica, 13, 465-473. Recuperado em 27 de setembro de 2017, de http://www. scielo.br/pdf/prc/v13n3/v13n3a14.pdf

Csikszentmihalyi, M. (1990). Flow: The psychology of optimal experience. New York, NY: Harper \& Row.

Csikszentmihalyi, M. (2004). What we must accomplish in the coming decades. Journal of Religion \& Science, 39(2), 359-366. DOI: 10.1111/j.1467-9744.2004.00579. x.

Diener, E., Suh, E. M., Lucas, R. E., \& Smith, H. L. (1999). Subjective Well-Being: Three Decades of Progress. Psychological Bulletin, 125(2), 276-302. DOI: https://doi.org/10.1037/0033-2909.125.2.276.

Diener, E., Wirtz, D., Tov, W., Kim-Prieto, C., Choi, D., Oishi, S., \& Biswas-Diener, R. (2009). New measures of wellbeing: Flourishing and positive and negative feelings. Social Indicators Research, 39, 247-266.

Dik, B. J., Duffy, R. D., Allan, B. A., O’Donnell, M, B., Shim, Y, \& Steger, M. F. (2015). Purpose and Meaning in Career Development. Applications. The Counseling Psychologist, 43(4), 558-585. DOI: 10.1177/0011000014546872.

Duarte, M. E. (2017). Counselling and well-being: on the road to realities. British Journal of Guidance \& Counselling, 1-11. DOI: 10.1080/03069885.2017.1309641. 
Erikson, E. H. (1968). Identity Youth and crisis. New York: W.W. Norton \& Company.

Fonseca, P. N., Nascimento, B. S., Barbosa, L. H. G. M., Vione, K. C., \& Gouveia, V.V. (2015). Flourishing Scale: Evidence of Its Suitability to the Brazilian Context. Social Inquiry into Well-Being, 1(2), 33-40. DOI: 10.13165/SIIW-15-1-2-07.

Formiga, N. S. (2010). Escala de exigência e responsividade parental: Evidência da estrutura fatorial em jovens da Paraíba, Brasil. Psicologia Argumento., Curitiba, 28(62), 209-224.

Keyes, C. L. M. (2002). The mental health continuum: From languishing to flourishing in life. Journal of Health and Social Behavior, 43, 207-222. DOI: 10.2307/3090197.

Granado, J. I., Santos, A. A., Almeida, L. S., Soares, A. P.,, \& Guisande, M. A. (2005). Integração académica de estudantes universitários: Contributos para a adaptação e validação do QVA no Brasil. Psicologia e Educação, 4(2), 31-41.

Gysbers, N. C., Heppner, M, J., \& Johnston, J. A. (2014). Career counselling: holism, diversity, and strengths. $\left(4^{\text {th }}\right)$. Alexandria: American Counseling Association.

Hill, N. E., \& Wang, M-T. (2015). From middle school to college: Developing aspirations, promoting engagement, and indirect pathways from parenting to post high school enrollment. Developmental Psychology, 51(2), 224-235. DOI:10.1037/a003836710.1037/a0038367.

Hofmans, J., Dries, N., \& Pepermans, R. (2008). The Career Satisfaction Scale: Response bias among men and women. Journal of Vocational Behavior, 73(3), 397-403. DOI: 10.1016/j.jvb.2008.08.001.

Holland, J. L. (1997). Making vocational choices: A theory of vocational personalities and work environments ( $3^{\text {rd }}$ ed.). Odessa, FL: Psychological Assessment Resources.

Hutz, C. S. \& Bardagi, M. P. (2006). Indecisão profissional, ansiedade e depressão na adolescência: a influência dos estilos parentais. Psico-USF, 11(1), 65-73.

Lamborn, S. D. (1991). Patterns of competence and adjustment among adolescents from authoritative, authoritarian, indulgent, and neglectful families. Child Development, 62, 1049-1065. DOI: 10.1111/j.1467-8624. 1991.tb01588. x.

Laranjeira, M. \& Teixeira, M.O. (2016). Perceção de feedback d0 professor e autoconceito vocacional. Revista Brasileira de Orientação Profissional,17(2), 211-223.

Lent, R. W., \& Brown, S. D. (2006). Integrating person and situation perspectives on work satisfaction: A social-cognitive view. Journal of Vocational Behavior, 69, 236-247. DOI: 10.1016/j.jvb.2006.02.006.

Lent, R. W., Brown, S. D., \& Hackett, G. (1994). Toward a unifying social cognitive theory of career and academic interest, choice, and performance. Journal of Vocational Behavior, 45, 79-122. DOI: 10.1006/jvbe.1994.1027.

Lent, R. W., Singley, D., Sheu, H., Gainor, K. A, Brenner, B. R., Treistman, D., \& Ades, L. (2005). Social Cognitive Predictors of Domain and Life Satisfaction: Exploring the Theoretical Precursors of Subjective Well-Being. Journal of Counseling Psychology, 5(3), 429-442. DOI:10.1037/0022-0167.52.3.429.

Lent, R. W., Taveira, M.C., \& Costa-Lobo, C. (2012). Two tests of the social cognitive model of well-being in Portuguese college students. Journal of Vocational Behavior, 80(2), 362-371. DOI:10.1177/1069072716657821.

Lopes, A. R. \& Teixeira, M. O. (2012). Projetos de carreira, autoeficácia e sucesso escolar em ambiente multicultural. Revista Brasileira de Orientação Profissional, 13(1), 7-14. Recuperado em 27 de setembro de 2017, de http://pepsic. bvsalud.org/pdf/rbop/v13n1/03.pdf

Maroco, J. \& Garcia-Marques, T. (2006). Qual a fiabilidade do alfa de Cronbach? Questões antigas e soluções modernas? Laboratório de Psicologia, 4(1), 65-90.

McCrae, R. R., \& Costa, P. T., Jr. (1996). Toward a new generation of personality theories: Theoretical contexts for the five-factor model. In J. S. Wiggins (Ed.), The five-factor model of personality: Theoretical perspectives, (p. 51-87). New York: Guilford.

Myers, J. E., Sweeney, T. J., \& Witmer, J. J. (2000). The Wheel of Wellness Counseling for Wellness: A Holistic Model for Treatment Planning. Journal of Counseling \& Development, 78, 251-266.

Ntalianis, F. (2010). Do personality and learning climate predict competence for learning? An Investigation in a Greek academic setting. Learning and Individual Differences, 20, 664-668. DOI: 10.1016/j.lindif.2010.08.003.

Ojeda, L., Navarro, R., L., \& Flores, L., Y. (2011). Social Cognitive Predictors of Mexican American College Student's Academic and Life Satisfaction. Journal of Counseling Psychology, 58(1), 61-71. DOI: 10.1037/a0021687.

Rammstedt, B. \& Jonh, O. (2007). Mesuring personality in one minute or less: A 10-item short version of the Big Five Inventory in English and German. Journal of Research in Personality, 41, 203-212. DOI: 10.1016/j.jrp.2006.02.001.

Ramos, L. M. A. (2016). Os mecanismos sócio-cognitivos e o bem-estar psicológico: teste de um modelo integrativo em estudantes universitários. Dissertação de doutoramento não publicada. Universidade da Beira Interior, Covilhã.

Ramos, L. A., Paixão, M., P., \& Simões, M., F., (2011). Os Mecanismos sociocognitivos e o bem-estar psicológico: Proposta de um modelo Integrativo. Revista Portuguesa de Pedagogia. 45(2), 133-147. DOI: 10.14195/1647-8614_45-2_6. 
Robertson, P. J. (2015). Positive psychology: A movement to reintegrate career counselling within counselling psychology? Counselling Psychology Review, 30(3), 26-36.

Rogers, C. (1961). On becoming a person: A therapist's view of psychotherapy. Boston, USA: Houghton Mifflin.

Rogers, M., Creed, P., \& Searle, J. (2012). Person and environmental factors associated with well-being in medical students. Personality and Individual Differences, 52, 472-477. DOI: 10.1016/j.paid.2011.11.006.

Savickas, M. L. (2013). Career construction theory and practice. In R. W. Lent \& S. D. Brown (Eds.), Career development and counseling: Putting theory and research to work ( $2^{\text {nd }}$ ed., pp. 144-180). Hoboken, NJ: John Wiley.

Sbicigo, J. B., Teixeira, M. A. P., Dias, A. C. G., \& DelÁglio, D. D. (2012). Propriedades Psicométricas da Escala de Autoeficácia Geral Percebida (EAGP). Psico, 43(2), 139-146.

Schwarzer, R. (2017). The General Self-Efficacy Scale (GSE). https://userpage.fu-berlin.de/health/engscal.htm.

Schwarzer, R. \& Jerusalem, M. (1995). Generalized Self-Efficacy scale. In J. Weinman, S. Wright, \& M. Johnston, Measures in health psychology: A user's portfolio. Causal and control beliefs (pp. 35-37). Windsor, UK: Nfer-Nelson.

Seligman, M. E. P. (2011). Flourish: A visionary new understanding of happiness and well-being. New York, NY: Free Press.

Seligman, M. E. P. \& Csikszentmihalyi, M. (2000). Positive psychology. An introduction. American Psychologist, 55, 5-14. DOI: 10.1037/0003-066X.55.1.5.

Spokane, A. R., Meir, E. L., \& Catalano, M. (2000). Person-Environment congruence and Holland's theory: A review and reconsideration. Journal of Vocational Behavior, 57(2), 137-187.

Steele, C., Andrews, H., \& Upton. D. (2012). Statistics in psychology. Harlow: Pearson Education.

Super, D. E. (1990). A life-span, life-space approach to career development. In D. Brown \& L. Brooks (Eds.), Career choice and development: Applying contemporary theories to practice (2 $2^{\text {nd }}$ ed., pp. 197-261). San Francisco: Jossey-Bass.

Teixeira, M. A. P. \& Gomes, W. B. (2004). Estou-me formando... e agora? Reflexões e perspectivas de jovens formandos universitários. Revista Brasileira de Orientação Profissional, 5(1), 47-52. Recuperado em 27 de setembro de 2017, de http://pepsic.bvsalud.org/pdf/rbop/v5n1/v5n1a05.pdf

Teixeira, M. O. \& Bardagi, M. (2016). Relationship between parenting styles and career development variables in Portuguese students. In Tamara Ramiro-Sánchez \& M Ma Teresa Ramiro (Coord.). Avances enciencias de la educación $y$ deldesarrollo, 2015, (1080-1086). Asociación Española de Psicología Conductual (AEPC). Recuperado: http:// congresoeducacion.es/edu_web4/capitulos_2015.pdf.

Teixeira, M.A.P., Bardagi, M.P. \& Gomes, W. B. (2004). Refinamento de um instrumento para avaliar responsividade e exigência parental percebidas na adolescência. Avaliação Psicológica, 3(1), 1-12. Universidade Federal do Rio Grande do Sul. Rio Grande do Sul.

Teixeira, M.O., \& Bardagi, M. P. (2016, setembro). Estudo intercultural dos Estilos de Parentalidade. Comunicação apresentada no Simpósio Contextos de desenvolvimento da carreira e de vida. Comunicação no $3^{\circ}$ Congresso da Ordem dos Psicólogos Portugueses. Porto.

Teixeira, M. O. \& Ferreira, I. A. (no prelo). Fatores familiares e escolares nas aspirações, na autoeficácia e no sucesso académico: um estudo exploratório com estudantes de $9^{\circ}$ ano. Revista Portuguesa de Psicologia.

Teixeira, M.O. \& Laranjeira, M. (2016, setembro). Autoeficácia para o desenvolvimento da carreira: efeitos dos contextos familiares, sociais e académicos. Comunicação apresentada no Simpósio Contextos de desenvolvimento da carreira e de vida. Comunicação no $3^{\circ}$ Congresso da Ordem dos Psicólogos Portugueses. Porto.

Verducci, S., \& Gardner, H. (2006). Good work: its nature, its nurture. In F. A. Huppert, N. Baylis, \& B. Keverne. The science of well-being (2 $2^{\text {nd }}$ Ed.). (pp. 343-360). Oxford: Oxford University Press.

Zunker, V. (2008). Career, work and mental health. Integrating career and personal counseling. London: Sage.

Recebido: 14/10/2016

$1^{a}$ reformulação: 22/07/2017

$2^{a}$ reformulação: 02/09/2017

Aceite final: 26/09/2017

Sobre as autoras

Maria Odília Teixeira é Professora Auxiliar da Faculdade de Psicologia da Universidade de Lisboa. Especialista no domínio do aconselhamento de carreira, com diversas publicações neste campo.

Cátia João Costa é Mestre em Psicologia Clinica Aplicada. Psicóloga Clínica com funções na Santa Casa da Misericórdia de Porto de Mós. 